\title{
Estimating Testing Time Extension Ratios for Students with Disabilities from Item Cumulative Curves
}

\author{
Mamoru Fujiyoshi ${ }^{1}$ and Akio Fujiyoshi ${ }^{2}$ \\ 1 Research Division, National Center for University Entrance Examinations, \\ 2-19-23 Komaba, Meguro-ku, Tokyo 153-8501, Japan \\ 2 Department of Computer and Information Sciences, Ibaraki University, 4-12-1 \\ Nakanarusawa, Hitachi, Ibaraki 316-8511, Japan
}

Summary. This study was conducted to define item cumulative score ratio curves and item cumulative completion ratio curves and to propose a new method to estimate fair extension ratios of testing time for students with disabilities from these curves. The proposed method estimates extension ratios of testing time of regular tests under the time-limit method from experimentally collected test data utilizing the work-limit method. In this experiment we found that for students with visual disabilities, the present extension ratio of testing time at the National Center Test for University Admissions in Japan is fair in Mathematics and English and that in order to improve the present testing method of the National Center Test, further extension of testing time is necessary for some subjects with a large amount of reading questions, such as Japanese.

Key words: Student with disabilities, Testing time, Item cumulative curve, Subject cumulative curve, Computer-based test

\section{Introduction}

The purpose of this study is to develop a new estimation method of fair extension ratios of testing time for students with disabilities in the National Center Test for University Admissions. The National Center Test is the joint first stage achievement test for admissions of all national and local public universities as well as some private universities in Japan. Every year, about 600,000 students take it. As for disabled candidates, various kinds of special treatment have been administered (Fujiyoshi 1995).

The extension of testing time has been administered for disabled students according to the type and degree of their disabilities in order to eliminate the reflection of the individual's impairment on assessment of achievement level or aptitude (Fujiyoshi 1995; Laing and Farmer 1984; Willingham et al. 1988). The present extension ratio of testing time at the National Center Test is 1.3 or 1.5, and the extension ratios of the ACT Assessment and the Scholastic 
Aptitude Test in the U.S. are from 1.8 to 4.8 (Bennett et al. 1985; Fujiyoshi 1995, 1997, 1999; Packer 1987; Ragosta and Wendler 1992; Willingham et al. 1988). However, the ground of the extension ratio of testing time has long been based only on experience and lacked quantitative explanations. It has been thought that multivariate or stochastic analysis cannot be used because the number of candidates with disabilities is so few. It has also been believed that defining fairness in extension ratios of testing time is delicate issue because the distributions of achievement level or aptitude for disabled students and nondisabled students might be different.

Recently, an estimation method comparing the distributions of completion time of nondisabled subjects and disabled subjects was developed (Fujiyoshi 1997; Packer 1987; Ragosta and Wendler 1992). In the estimation procedure of this method, subject cumulative completion ratio curves are employed. Another estimation method comparing the distributions of completion time weighted by score was also proposed (Fujiyoshi 1999). This method employs subject cumulative score ratio curves. Because the type and degree of disabilities of disabled students are multifarious and the number of disabled candidates is very small, it is still questionable whether the estimated extension ratios from these methods are fair.

In this study item cumulative score ratio curves and item cumulative completion ratio curves are re-defined (Fujiyoshi et al. 2001), and a new method that enables the estimation of fair extension ratios even when applied to a small number of subjects with disabilities is proposed. We also report the estimated extension ratios of testing time calculated through the method for partially sighted students who use large print test booklets and for blind students who use braille format test booklets.

\section{The Definitions of Subject Cumulative Curves and Item Cumulative Curves}

In this section we will mathematically re-define the two types of subject cumulative curves and the two types of item cumulative curves.

Suppose that there are $n$ subjects $\left(\right.$ subject $_{1}$, subject $_{2}, \ldots$, subject $\left._{n}\right)$ and $m$ items $\left(\right.$ item $_{1}$, item $_{2}, \ldots$, item $\left.{ }_{m}\right)$. For each item $_{1}$, item $_{2}, \ldots$, item $_{m}$, suppose also that the allotment of item $_{j}$ is allotment ${ }_{j}$. Let $t_{i, j}$ be the time that subject $_{i}$ completed the answer of item $_{j}$. If subject $_{i}$ leaves item $_{j}$ unanswered, let $t_{i, j}=0$. Let $t_{i}$ be $\max \left\{t_{i, j} \mid j=1, \ldots, m\right\}$. Let $c_{i, j}$ be an integer such that if subject ${ }_{i}$ answered item $_{j}$ correctly, $c_{i, j}=1$, otherwise $c_{i, j}=0$. Let total be $\sum_{i=1}^{n} \sum_{j=1}^{m} c_{i, j} \cdot$ allotment $_{j}$.

\subsection{Subject Cumulative Curves}

Definition 2.1. The subject completion function for subject $_{i}, S C_{i}$ is a function such that if $t<t_{i}$, then $S C_{i}(t)=0$, otherwise $t \geq t_{i}$, then $S C_{i}(t)=1$. 
Definition 2.2. The distribution function of frequency of completed subjects $F C S$ and the distribution function of score of completed subjects $S C S$ are defined as follows:

$$
\begin{gathered}
F C S(t)=\sum_{i=1}^{n}(1 / n) \cdot S C_{i}(t) \\
S C S(t)=\sum_{i=1}^{n}\left(\sum_{j=1}^{m} c_{i, j} \cdot \text { allotment }_{j} / \text { total }\right) \cdot S C_{i}(t)
\end{gathered}
$$

Definition 2.3. A subject cumulative completion ratio curve is a set of points on a coordinate system with time $t$ on the horizontal axis and cumulative relative frequency of completed subjects $F C S(t)$ on the vertical axis (Fujiyoshi 1997). A subject cumulative score ratio curve is a set of points on coordinate system with time $t$ on the horizontal axis and cumulative relative score of completed subjects $S C S(t)$ on the vertical axis (Fujiyoshi 1999).

\subsection{Item Cumulative Curves}

Definition 2.4. The item completion function for subject $_{i}$ and $i t e m_{j}, I C_{i, j}$ is a function such that if $t<t_{i, j}$, then $I C_{i, j}(t)=0$, otherwise $t \geq t_{i, j}$, then $I C_{i, j}(t)=1$.

Definition 2.5. The distribution function of frequency of completed items $F C I$ and the distribution function of score of completed items $S C I$ are defined as follows:

$$
\begin{gathered}
F C I(t)=\sum_{i=1}^{n} \sum_{j=1}^{m}(1 / m n) \cdot I C_{i, j}(t) \\
S C I(t)=\sum_{i=1}^{n} \sum_{j=1}^{m}\left(c_{i, j} \cdot \text { allotment }_{j} / \text { total }\right) \cdot I C_{i, j}(t)
\end{gathered}
$$

Definition 2.6. An item cumulative completion ratio curve is a set of points on a coordinate system with time $t$ on the horizontal axis and cumulative relative frequency of completed items $F C I(t)$ on the vertical axis. An item cumulative score ratio curve is a set of points on coordinate system with time $t$ on the horizontal axis and cumulative relative score of completed items $S C I(t)$ on the vertical axis (Fujiyoshi et al. 2001).

\section{Experimentation on the Estimation of Testing Time Extension Ratios}

\subsection{Purpose}

By analyzing test data acquired in this experiment, we will estimate testing time extension ratios for students with disabilities from subject cumulative completion ratio curves, subject cumulative score ratio curves, item cumulative completion ratio curves and item cumulative score ratio curves. 


\subsection{Method}

We developed the computer-based test (CBT) to record the answering process of a conventional paper-and-pencil test (PPT) because the National Center Test is a conventional PPT which uses optical readable marking sheets (Fujiyoshi et al. 2001). Employing a pen computer (Amity SV, Mitsubishi Electric Corporation), the CBT was designed to simulate the answering process of the PPT as faithfully as possible. The PPT sheet is displayed on the computer screen, and the electronic pen replaces the pencil.

Subjects are freshmen and sophomores of four-year universities. The nondisabled group consisted of 99 students. The partially sighted group consists of 8 students who use large print test booklets. Their corrected visual acuity of common eyesight is from 0.01 to 0.1 , and its median is 0.1 . The blind group included 17 students who used braille format test booklets.

Nondisabled students took the CBT. On the other hand, partially sighted students were given large print test booklets and blind subjects were given braille format test booklets. The behavior of disabled students was observed by test monitors, and the answering process of disabled students was input by the monitors into the recording system used on the CBT.

The test sets were prepared from questions previously used in the National Center Test. Calculating from the quantity of exam questions, testing time required for each of the three subject was 40 minutes.

The test procedure started with issuing instructions; after that the subjects answered test questions in accordance with the work-limit method.

\subsection{Results}

Score Distributions: Fig. 1 is Box-and-whisker plots representing the score distributions of the three groups for Japanese, Mathematics and English. The vertical lines in the middle of the box plots indicate the medians. The ' + ' symbols in the boxes are the means.

The score distributions are almost similar among the three groups for each of the three subjects. Though the median of the nondisabled group is from 3 to 28 points (out of 80 points) higher than that of the partially sighted group and the blind group, there are no significant differences among the medians of the three groups for each of the three subjects according to the Mann-Whitney test.

Item Cumulative Score Ratio Curves: Firstly, we drew item cumulative score ratio curves from the experimental test data. Fig. 2 shows the curves for Japanese. The left curve made up of ' $x$ ' symbols shows the curve for the nondisabled group. The thin line is the curve of the Compound Weibull distribution function, which is used for smoothing and can be expressed by a simple formulation. The middle curve made up of ' $*$ ' shows the curve for the partially sighted group. The bold line is the curve of the Compound Weibull 


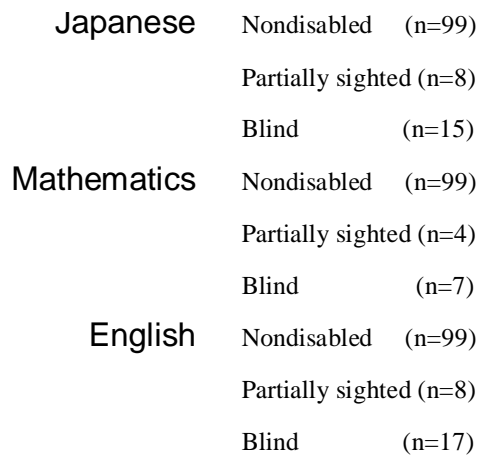
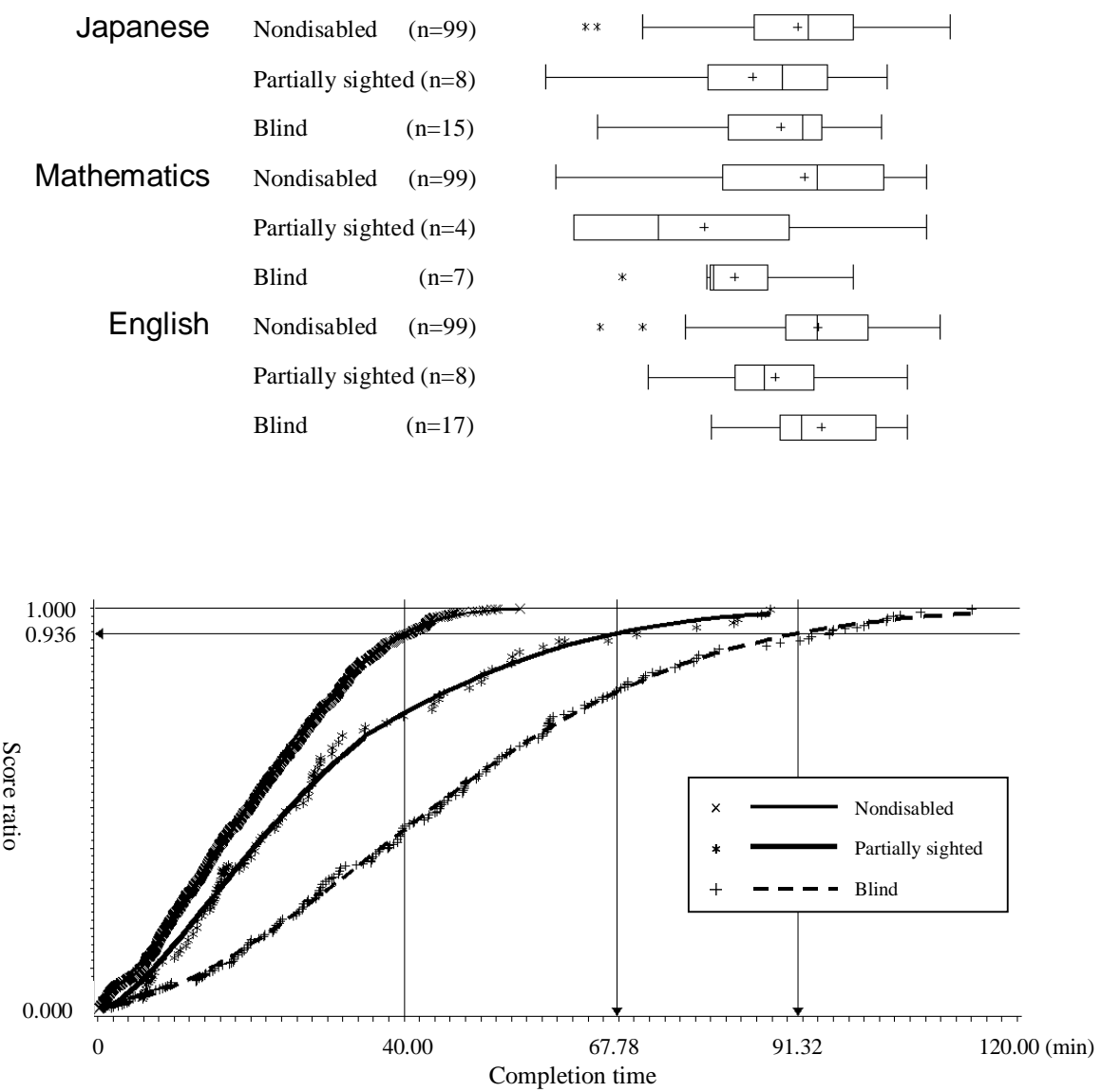

Fig. 2. Item cumulative score ratio curves for Japanese

distribution function. The right curve made up of ' + ' shows the curve for the blind group. The broken line is the curve of the Compound Weibull distribution function.

Author's Policy and Estimation Method: The author's policy of the estimation of extension ratio of testing time is to guarantee the partially sighted group and the blind group sufficient testing time that enables them to arrive at the same score ratio or completion ratio of the nondisabled group at the time limit of the regular tests.

Based on the Japanese curves in Fig. 2, we will show how to estimate the extension ratios of testing time from item cumulative score ratio curves. In order to formulate the estimation process of extension ratios, the curves of the compound Weibull distribution function are used instead of the item cumulative curves themselves. 
We first checked the score ratio of the nondisabled group at the time limit of 40 minutes. In the case of Japanese, the score ratio to guarantee is 0.936, that is, the vertical coordinate of the intersection of the nondisabled group's curve and the vertical line of 40 minutes.

Next, we found the horizontal coordinates of the intersection of the horizontal line of 0.936 and the disabled group's curves. The completion time is 40 minutes for the nondisabled group, 67.78 minutes for the partially sighted group and 91.32 minutes for the blind group.

The estimation value of extension ratio of testing time is defined as the ratio of the completion time for the disabled group to completion time for the nondisabled group at the score ratio to guarantee. In the case of Japanese, the extension ratios of testing time are estimated to be $1.696(67.78 / 40.00)$ for the partially sighted group and $2.284(91.32 / 40.00)$ for the blind group.

Estimated Extension Ratios of Testing Time: We estimated the extension ratios of testing time for the disabled groups by means of the following four estimation methods:

(Method 1) Estimation from item cumulative score ratio curves: This method is explained in Section 3.3.3.

(Method 2) Estimation from item cumulative completion ratio curves: This method is obtained from Method 1 exchanging score ratio for completion ratio.

(Method 3) Estimation from subject cumulative score ratio curves: This method is obtained from Method 1 exchanging item cumulative curves for subject cumulative curves; this method was introduced in (Fujiyoshi 1999).

(Method 4) Estimation from subject cumulative completion ratio curves: This method is obtained from Method 3 exchanging score ratio for completion ratio; this method was introduced in (Fujiyoshi 1997).

Table 1 shows the score ratios (Method 1 and 3) and the completion ratios (Method 2 and 4 ) of the nondisabled group at 40 minutes. Table 2 shows the completion time and the extension ratios estimated from the item cumulative score ratio curves (Method 1), the item cumulative completion ratio curves (Method 2), the subject cumulative score ratio curves (Method 3) (Fujiyoshi 1999), and the subject cumulative completion ratio curves (Method 4) (Fujiyoshi 1997).

The estimated extension ratios from the four methods are similar.

Table 1. Score ratios (Method 1 and 3) and completion ratios (Method 2 and 4)

\begin{tabular}{|c|c|c|c|c|}
\hline & Method & Method & Tethod & Method 4 \\
\hline$\overline{\text { Japs }}$ & 0.936 & 0.937 & 0.690 & 0.693 \\
\hline Mathematics & 0.920 & 0.888 & 0.549 & 0.549 \\
\hline English & 0.942 & 0.953 & 0.664 & 0.664 \\
\hline
\end{tabular}


Table 2. Completion times and estimated extension ratios from the four methods

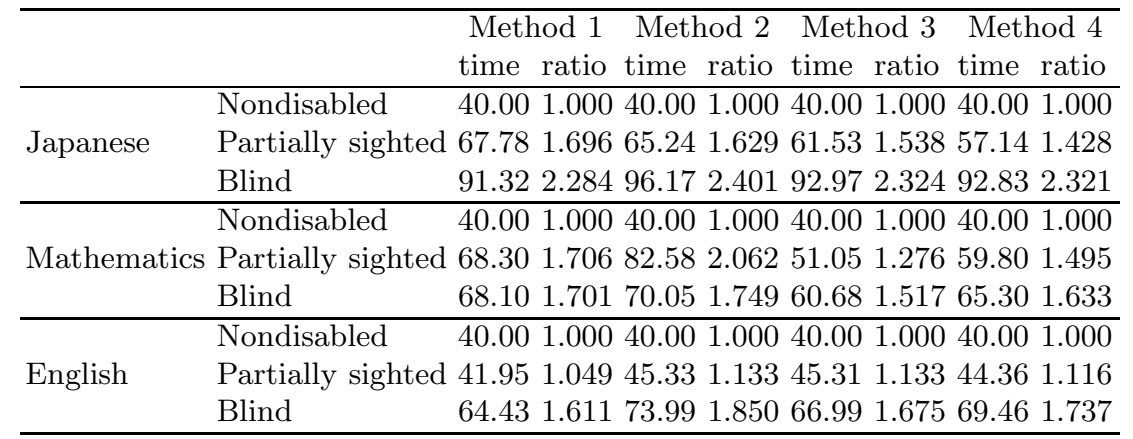

\subsection{Discussion}

We estimated extension ratios of testing time for students with disabilities from the four methods using the test data acquired in this experiment. In the National Center Test for University Admissions, the present extension ratio of testing time for all subjects has been 1.3 for partially sighted students whose corrected visual acuity of common eyesight is less than 0.15 since 1988. For blind students, the present extension ratio has been 1.5 since 1979 .

The estimated extension ratios of testing time for disabled students are almost the same as the present extension ratio for Mathematics and English. However, the estimated extension ratios for Japanese are much greater than the present extension ratio.

For Mathematics, the estimated extension ratio for the partially sighted group seems much greater than the present extension ratio, 1.3. However, it is not a reliable figure because the number of subjects was too small.

\section{Conclusion}

We proposed methods to estimate fair extension ratios of testing time for disabled students according to author's policy of fairness from the item cumulative score ratio curves and the item cumulative completion ratio curves. It was experimentally shown that the extension ratios for disabled students in regular tests under the time-limit method can be estimated from the experimental test data under the work-limit method.

Though the estimated extension ratios from the four methods are similar, we think that the estimation method by item cumulative score ratio curves surpasses the others because of the following features in methodology:

Firstly, as item cumulative curves have more measured points than subject cumulative curves, item cumulative curves are smooth and stable even with a 
small number of subjects. Secondly, the estimation method by score ratio can be applied even if the score distributions of two groups are different because the estimation is made from the distributions of completion time weighted by score. Thirdly, the estimation of extension ratios of testing time is fair because sufficient testing time is guaranteed for disabled students to arrive at the same score ratio of nondisabled students at the time limit of regular tests.

This study revealed that the estimated extension ratios of testing time for Mathematics and English for students with visual disabilities are almost the same as the present extension ratios of the National Center Test. This means that the present extension ratios of testing time are fair in Mathematics and English. In order to improve the present testing method of the National Center Test, further extension of testing time is necessary for some subjects which include a large amount of reading questions, such as Japanese.

\section{References}

Bennett, R. E., Rock, D. A., and Kaplan, B. A. (1985) The Psychometric Characteristics of the SAT for Nine Handicapped Groups. ETS Research Report RR85-49, 1-70.

Fujiyoshi, M. (1995) Relationship of Type of Disability and Examination Method to Distribution of Scores of Students with Disabilities on the Joint First Stage Achievement Tests (in Japanese). Japanese Journal of Special Education, 33(2), 61-70

Fujiyoshi, M. (1997) A New Method for Estimation the Time to Be Extended for Testing Students with Visual Disabilities from the Response Time Curves (in Japanese). The National Center for University Entrance Examinations Research Bulletin, No. 27, 1-18

Fujiyoshi, M. (1999) Improvement for Estimation Method of Amount of Testing Time Extension for Students with Visual Disabilities by Means of Time-Score Ratio Curves (in Japanese). Japanese Journal of University Entrance Examination Research, No. 9, 31-37

Fujiyoshi, M., Fujiyoshi, A., Ishizuka, T. (2001) Comparability of Paper-and-Pencil Test and Computer-Based Test in Terms of Distributions of Completion Time and Score. The National Center for University Entrance Examinations Research Bulletin, No. 30, 67-82

Laing, J., Farmer, M. (1984) Use of the ACT Assessment by Examinees with Disabilities. ACT Research Report, 84, 1-35

Packer, J. (1987) SAT Testing Time for Students with Disabilities. ETS Research Report, RR-87-37, 1-25

Ragosta, M., Wendler, C. (1992) Eligibility Issues and Comparable Time Limits for Disabled and Nondisabled SAT Examinees. ETS Research Report, RR-92-35, $1-33$

Willingham, W. W., Ragosta, M., Bennett, R. E., Braun, H., Rock, D. A., Powers, D. E. (1988) Testing Handicapped People. Allyn and Bacon, Inc., Massachusetts 